\title{
AN EXPANSION OF BIVARIATE SPLINE FUNCTIONS
}

\author{
HUAN-WEN LIU ${ }^{1}$
}

(Received 25 July 1997; revised 13 January 1998)

\begin{abstract}
Let $\Delta$ denote a triangulation of a planar polygon $\Omega$. For any positive integer $0 \leq r<k$, let $S_{k}^{r}(\Delta)$ denote the vector space of functions in $C^{r}$ whose restrictions to each triangle of $\Delta$ are polynomials of total degree at most $k$. Such spaces, called bivariate spline spaces, have many applications in surface fitting, scattered data interpolation, function approximation and numerical solutions of partial differential equations. An important problem is to give the function expression. In this paper, we prove that, if $(\Delta, \Omega)$ is type$X$, then any bivariate spline function in $S_{k}^{r}(\Delta)$ can be expressed by a series of univariate polynomials and a special bivariate finite element function in $S_{k-r}^{0}(\Delta)$ satisfying a so-called integral conformality condition system. We also give a direct sum decomposition of the space $S_{k}^{r}(\Delta)$. In addition, the dimension of $S_{r+1}^{r}(\Delta)$ for a kind of triangulation has been determined.
\end{abstract}

\section{Introduction}

For a connected polygonal region $\Omega$ in $\mathbf{R}^{2}$, let $\Delta$ be a triangulation of $\Omega$, and by this, we mean that the complement of $\Delta$ relative to $\Omega$ consists of a finite number of triangles such that none of the vertices of any triangle lies on the interior of any edge of another triangle. For $0 \leq r \leq k-1$, where $r$ and $k$ are integers, we define $S_{k}^{r}(\Delta)$ to be the vector space of $C^{r}$ functions which are piecewise polynomials with total degree at most $k$ over each triangle of $\Delta$. The space $S_{k}^{r}(\Delta)$ is called a bivariate spline space with degree $k$ and smoothness order $r$.

It is well-known that, in the one dimensional case, univariate spline functions are very useful in many practical applications. For example, the cubic spline, which has good smoothness and solves the optimal interpolation problem, is widely applied in solving bending problems of plates and shells and in the surface design of cars, ships and aeroplanes. In the early and mid seventies, because of the increasing

\footnotetext{
${ }^{1}$ School of Mathematics and Applied Statistics, The University of Wollongong, Wollongong, NSW 2522, Australia.

(C) Australian Mathematical Society 2000, Serial-fee code 0334-2700/00
} 
predominance of the finite element method, people began to study spline functions in two dimensions, see $[22,25,26]$. Since then, bivariate splines have been widely applied in multivariate function approximation, surface fitting and design, the finite element methods and numerical solutions of partial differential equations. In recent years, they have been used in scattered data interpolation $[1,8]$ and wavelets $[6,7]$.

With such a widespread range of practical applications, the space of bivariate spline functions is of great interest to many researchers. It is important to determine both the dimension of such a space and the expression of the function. Since Morgan and Scott gave the first result on dimension [22] in 1975, much work has been done in determining the spaces dimension, see $[2,3,8,13,21,24]$. As for research into the expression of bivariate spline functions, there are currently three main methods: Bernstein-Bézier representation (B-form, B-net) $[4,11,12,14,16]$, the basic spline (B-spline or box spline) method $[5,10,17]$ and the smoothing cofactor method $[8,9,25]$.

In $[19,20]$, a so-called integral representation of bivariate splines is proposed by the present author. This together with the B-net method, has been used to determine the dimension of the double periodic spline space $\tilde{S}_{k}^{1}\left(\triangle_{m n}^{(1)}\right)$ with $k \geq 4$ on Type-1 triangulation $\triangle_{m n}^{(1)}$ [19], the bivariate quadratic spline space $S_{2}^{1}(\Delta)$ [20] and the cubic spline space $S_{3}^{1}(\Delta)$ over stratified triangulation [21]. In this paper, an expansion of the bivariate spline function in $S_{k}^{r}(\Delta)$ is presented, a direct sum decomposition of $S_{k}^{r}(\Delta)$ is given and the dimension of $S_{r+1}^{r}(\Delta)$ for a kind of particular triangulation is determined.

\section{An integral representation of bivariate splines}

For any triangulation $\Delta$, denote

$$
\begin{aligned}
V_{I} & :=\text { the set of all the interior vertices of } \Delta, \\
V_{B} & :=\text { the set of all the boundary vertices of } \Delta, \\
E_{I} & :=\text { the set of all the interior edges of } \Delta, \\
E & :=\text { the set of all the edges of } \Delta, \\
V & :=V_{I}+V_{B} .
\end{aligned}
$$

We say $(\Omega, \Delta)$ is of type- $X$ provided that there exists a rectangular coordinate system $X O Y$ such that the slope of any edge in $E$ equals neither 0 nor $\infty$ and the number of intersection points of the boundary $\partial \Omega$ and any straight line which is parallel to the $X$-axis is no more than two. For example, $(\Omega, \Delta)$ is always type- $X$ for any triangulation $\Delta$ if $\Omega$ is a connected convex polygonal region. We will merely consider the case that $(\Omega, \Delta)$ is type- $X$ throughout this paper. 


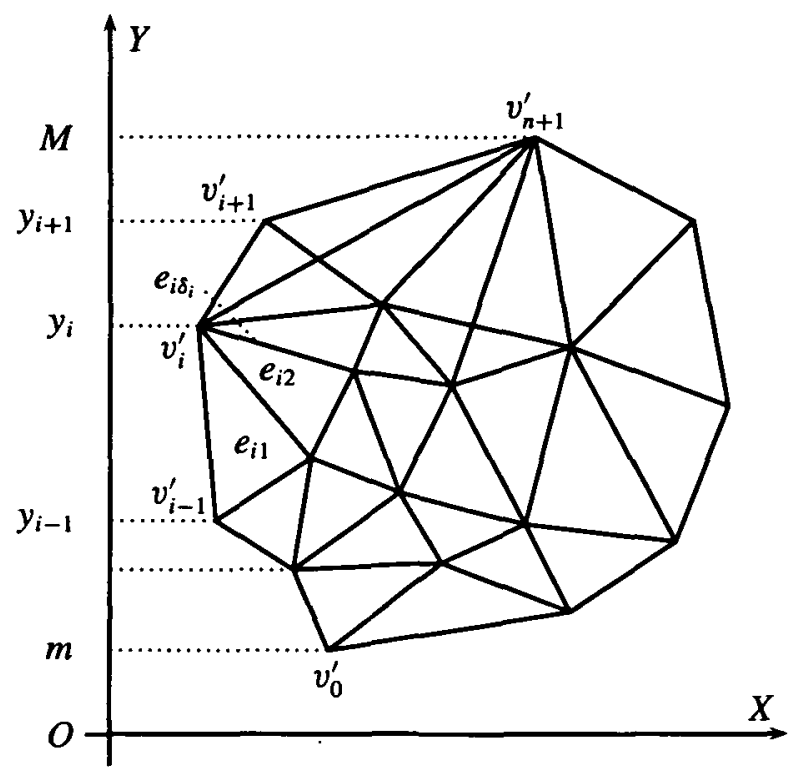

FIGURE 1. Planar polygon $\Omega$ and its triangulation $\Delta$.

We set up a rectangular coordinate system $X O Y$ as required above. Clearly the two boundary vertices of $\Delta$ with maximum and minimum ordinates divide $\partial \Omega$ into two parts. The left part is denoted by $\partial^{-} \Omega$ and the set of all the vertices on $\partial^{-} \Omega$ except for the end points is denoted by $V_{B}^{-}$.

For each $v \in V_{I}$, the union of all the triangles with the common vertex $v$ is called a standard cell with interior vertex $v$ and is denoted by $\Delta_{v}$. We denote the boundary vertices in $\Delta_{v}$, in the counter-clockwise direction, by $v_{j}, j=1,2, \ldots, d_{v}$, where $d_{v}$ is the number of edges emanating from $v$, called the degree of $v$. Set $v_{0}:=v, e_{j}:=v_{0} v_{j}$ and $k_{j}:=\operatorname{slope}\left(e_{j}\right)$. Clearly, $e_{j}$ can be described by the equation $x=l_{j}(y)$, where $l_{j}(y):=x_{0}+\left(y-y_{0}\right) / k_{j}$, and $\left(x_{0}, y_{0}\right)$ are the coordinates of $v_{0}$. In addition, the triangle between $e_{j}$ and $e_{j+1}$ is denoted by $T_{j}, j=1,2, \ldots, d_{v}$.

As shown in Figure 1, from bottom to top, we label consecutively all the vertices on $\partial^{-} \Omega v_{i}^{\prime}, i=0,1, \ldots, n, n+1$. And for $i=1, \ldots, n$, we number all the edges attached to $v_{i}^{\prime}$ in the counter-clockwise direction and label them $e_{i j}^{\prime}, j=1, \ldots, d_{i}^{\prime}$, where we have put $e_{i 1}^{\prime}:=v_{i-1}^{\prime} v_{i}^{\prime}$. Note that, with the above-mentioned assumption of slope $(e) \neq 0, \infty$ for any $e \in E$, the equation of $e_{i j}^{\prime}$ can be denoted by $x=\phi_{i j}(y)$, $i=1, \ldots, n, j=1, \ldots, d_{i}^{\prime}$. In addition, we let $T_{i j}^{\prime}$ stand for the triangle between $e_{i j}^{\prime}$ and $e_{i j+1}^{\prime}$.

From the assumption that $(\Omega, \Delta)$ is type- $X$, we can see easily that the projections of all vertices $v_{i}^{\prime}(i=0,1, \ldots, n, n+1)$ in the $Y$-axis form a univariate partition $\Delta_{y}$ 
of interval $[m, M]$ as follows:

$$
\Delta_{y}: \quad m=y_{0}^{\prime}<y_{1}^{\prime}<\cdots<y_{n}^{\prime}<y_{n+1}^{\prime}=M,
$$

where $y_{i}^{\prime}$ is the ordinate of $v_{i}^{\prime}$. We denote the equation of the broken line on which $\partial^{-} \Omega$ lies by $x=\Phi(y)$ with

$$
\Phi(y):= \begin{cases}\phi_{i 1}(y), & y \in\left[y_{i-1}^{\prime}, y_{i}^{\prime}\right], \quad i=1, \ldots, n \\ \phi_{n d_{n}^{\prime}}(y), & y \in\left[y_{n}, y_{n+1}^{\prime}\right] .\end{cases}
$$

For $0 \leq r \leq k-1$, where $r$ and $k$ are integers, we define $S_{k}^{r}(\Delta)$ to be the vector space of $C^{r}$ functions which are piecewise polynomials with total degree at most $k$ over each triangle of $\triangle$.

Let $\mathbf{P}_{k}$ and $\Pi_{k}$ denote the spaces of univariate and bivariate polynomials with degree at most $k$, respectively. As is well known [8,9,25], a necessary and sufficient condition for $s \in S_{k}^{r}(\Delta)$ is that the following conformality condition

$$
\sum_{j=1}^{d_{v}} q_{j}(x, y)\left[x-l_{j}(y)\right]^{r+1} \equiv 0
$$

holds for any $v \in V_{I}$, where $q_{j} \in \Pi_{k-r-1}$ is the smoothing cofactor of $s$ from $T_{j-1}$ to $T_{j}$ across edge $e_{j}$ around $v$ satisfying

$$
\left.s(x, y)\right|_{T_{j}}-\left.s(x, y)\right|_{T_{j-1}}=q_{j}(x, y)\left[x-l_{j}(y)\right]^{r+1} .
$$

Recently [20], another kind of identity, namely

$$
\sum_{j=1}^{d_{v}} \int_{l_{1}(y)}^{l_{j}(y)} q_{j}(x, y)\left[x-l_{j}(y)\right]^{r+1} d x \equiv 0,
$$

or

$$
\left.\sum_{j=1}^{d_{v}} \int_{l_{j}(y)}^{l_{j+1}(y)} s(x, y)\right|_{T_{j}} d x \equiv 0
$$

called the integral conformality condition of $s$ at the interior vertex $v$, was introduced and a kind of relevant spline space $\hat{S}_{k}^{r}(\Delta)$ was defined to be composed of $s$ such that

(i) $s \in S_{k}^{r}(\Delta)$;

(ii) the integral conformality condition (4) holds at each $v \in V_{I}$.

On the basis of this ([20] also see [21]), we obtain the following lemma. 
LEMMA 1 . Let $1 \leq r \leq k-1$ and $(\Omega, \Delta)$ be type- $X$; then a necessary and sufficient condition for $s \in S_{k}^{r}(\Delta)$ is that $s$ can be expressed as

$$
s(x, y)=p(y)+\int_{\Phi(y)}^{x} \eta(\tau, y) d \tau,
$$

where $\eta(x, y) \in \hat{S}_{k-1}^{r-1}(\Delta)$ and $p(y)$ is a univariate piecewise polynomial with degree at most $k$ on $\Delta_{y}$ such that

$$
p_{i}(y)=p_{0}(y)+\left.\sum_{t=1}^{i} \sum_{j=1}^{d_{t}^{\prime}-1} \int_{\phi_{i j}}^{\phi_{i j+1}} \eta(x, y)\right|_{T_{i j}^{\prime}} d x, \quad i=1, \ldots, n,
$$

with $p_{i}(y):=\left.p(y)\right|_{\left[y_{i}^{\prime}, y_{i+1}^{\prime}\right]}, i=0,1, \ldots, n$.

For convenience we here recast Lemma 1 as follows.

LEMMA 2. Let $1 \leq r \leq k-1$ and $(\Omega, \Delta)$ be type- $X$; then a necessary and sufficient condition for $s \in S_{k}^{r}(\Delta)$ is that $s$ can be expressed as

$$
s(x, y)=p(y)+\int_{\Phi(y)}^{x} \eta(\tau, y) d \tau,
$$

where $\eta(x, y) \in \hat{S}_{k-1}^{r-1}(\Delta)$ and $p(y)$ is a univariate piecewise polynomial with degree at most $k$ on $\Delta_{y}$ such that

$$
p(y)=p_{0}(y)+\left.\sum_{i=1}^{n}\left(y-y_{t}^{\prime}\right)_{+}^{0} \sum_{j=1}^{d_{i}^{\prime}-1} \int_{\phi_{i j}}^{\phi_{i j+1}} \eta(x, y)\right|_{T_{i j}^{\prime}} d x,
$$

where

$$
\left(y-y_{t}^{\prime}\right)_{+}^{0}:= \begin{cases}1 & y>y_{t}^{\prime} \\ 0 & \text { otherwise }\end{cases}
$$

\section{An expansion of bivariate spline functions}

Before giving our result, we define an integral projection operator $\mathbf{I}_{i}: \mathbf{C}^{0}(\Omega) \rightarrow$ $\mathbf{C}^{0}[m, M], i=0,1, \ldots, r-1$. For any piecewise polynomial function $f(x, y)$ defined on $\Delta$ with global smoothness at least 0 on the region $\Omega$, denote

$$
\begin{aligned}
& \mathbf{I}_{0}[f]:=\left.\sum_{i=1}^{n}\left(y-y_{i}^{\prime}\right)_{+}^{0} \sum_{j=1}^{d_{i}^{\prime}-1} \int_{\phi_{i j}}^{\phi_{i+1}} f(x, y)\right|_{T_{i j}^{\prime}} d x, \\
& \mathbf{I}_{1}[f]:=\mathbf{I}_{0}\left[\int_{\Phi}^{x} f\left(\tau_{r}, y\right) d \tau_{r}\right], \\
& \mathbf{I}_{i}[f]:=\mathbf{I}_{0}\left[\int_{\Phi}^{x} \int_{\Phi}^{\tau_{r-i+1}} \cdots \int_{\Phi}^{\tau_{r-1}} f\left(\tau_{r}, y\right) d \tau_{r} \cdots d \tau_{r-i+2} d \tau_{r-i+1}\right], \quad i=2, \ldots, r-1 .
\end{aligned}
$$


THEOREM 1. Let $1 \leq r \leq k-1$ and $(\Omega, \Delta)$ be type- $X$; then a necessary and sufficient condition for $s \in S_{k}^{r}(\triangle)$ is that $s$ has the following expansion:

$$
\begin{aligned}
s(x, y)= & \sum_{j=0}^{r-1} \frac{1}{j !}\left(x-\phi_{11}(y)\right)^{j}\left(p_{0}^{(j)}(y)+\mathbf{I}_{r-1-j}\left[s^{(r)}\right]\right) \\
& +\int_{\Phi(y)}^{\tau_{0}} \int_{\Phi(y)}^{\tau_{1}} \cdots \int_{\Phi(y)}^{\tau_{r-1}} s^{(r)}\left(\tau_{r}, y\right) d \tau_{r} \cdots d \tau_{2} d \tau_{1},
\end{aligned}
$$

where $\tau_{0}=x, p_{0}^{(j)}(y)$ are arbitrary univariate polynomials in $\mathbf{P}_{k-j}, j=0,1, \ldots, r-$ 1 , and $s^{(r)}(x, y) \in S_{k-r}^{0}(\triangle)$ satisfies the following integral conformality condition system at each interior vertex $v \in V_{I}$ :

$$
\left\{\begin{array}{l}
\sum_{j=1}^{d_{v}} \int_{l_{l_{l}}}^{l_{j}} q_{j}^{(r)}(x, y)\left[x-l_{j}\right] d x \equiv 0 \\
\sum_{j=1}^{d_{v}} \int_{l_{1}}^{l_{j}} \int_{l_{j}}^{x} q_{j}^{(r)}\left(x_{1}, y\right)\left[x_{1}-l_{j}\right] d x_{1} d x \equiv 0 \\
\sum_{j=1}^{d_{v}} \int_{l_{1}}^{l_{j}} \int_{l_{j}}^{x} \int_{l_{j}}^{x_{1}} q_{j}^{(r)}\left(x_{2}, y\right)\left[x_{2}-l_{j}\right] d x_{2} d x_{1} d x \equiv 0 \\
\ldots \ldots \cdots \\
\sum_{j=1}^{d_{v}} \int_{l_{1}}^{l_{j}} \int_{l_{j}}^{x} \int_{l_{j}}^{x_{1}} \cdots \int_{l_{j}}^{x_{r-2}} q_{j}^{(r)}\left(x_{r-1}, y\right)\left[x_{r-1}-l_{j}\right] d x_{r-1} \cdots d x_{2} d x_{1} d x \equiv 0
\end{array}\right.
$$

with $q_{j}^{(r)} \in \Pi_{k-r}$ being the smoothing cofactor of $s^{(r)}(x, y)$ from $T_{j}$ to $T_{j+1}$ across edge $e_{j}$ around $v$.

PROOF. From Lemma 2, we can easily see that a necessary and sufficient condition for $s^{(0)}(x, y)=s(x, y) \in S_{k}^{r}(\Delta)$ is that the following recurrence expressions

$$
\begin{aligned}
& s^{(0)}(x, y)=p^{(0)}(y)+\int_{\Phi(y)}^{x} s^{(1)}\left(\tau_{1}, y\right) d \tau_{1}, \quad s^{(1)} \in \hat{S}_{k-1}^{r-1}(\Delta), \\
& s^{(1)}(x, y)=p^{(1)}(y)+\int_{\Phi(y)}^{x} s^{(2)}\left(\tau_{2}, y\right) d \tau_{2}, \quad s^{(2)} \in \hat{S}_{k-2}^{r-2}(\Delta), \\
& s^{(r-1)}(x, y)=p^{(r-1)}(y)+\int_{\Phi(y)}^{x} s^{(r)}\left(\tau_{r}, y\right) d \tau_{r}, \quad s^{(r)} \in \hat{S}_{k-r}^{0}(\Delta),
\end{aligned}
$$

hold, where

$$
p^{(i)}(y)=p_{0}^{(i)}(y)+\mathbf{I}_{0}\left[s^{(i+1)}\right], \quad i=0,1, \ldots, r-1,
$$


with $p_{0}^{(j)}$ being arbitrary univariate polynomials in $\mathbf{P}_{k-j}, j=0,1, \ldots, r-1$.

From (13)-(15) we have

$$
\begin{aligned}
s(x, y)= & \sum_{i=0}^{r-1} \frac{1}{i !}[x-\Phi(y)]^{i} p^{(i)}(y) \\
& +\int_{\Phi(y)}^{\tau_{0}} \int_{\Phi(y)}^{\tau_{1}} \cdots \int_{\Phi(y)}^{\tau_{r-1}} s^{(r)}\left(\tau_{r}, y\right) d \tau_{r} \cdots d \tau_{2} d \tau_{1},
\end{aligned}
$$

with $\tau_{0}=x$. From the recursions (13)-(15) and equality (16), we have, for $0 \leq i<$ $r-1$,

$$
\begin{aligned}
p^{(i)}(y) & =p_{0}^{(i)}(y)+\mathbf{I}_{0}\left[s^{(i+1)}\right] \\
& =p_{0}^{(i)}(y)+\mathbf{I}_{0}\left[p^{(i+1)}(y)+\int_{\Phi(y)}^{\tau_{i+1}} s^{(i+2)}\left(\tau_{i+2}, y\right) d \tau_{i+2}\right] \\
& =p_{0}^{(i)}(y)+\sum_{t=1}^{n}\left(y-y_{t}^{\prime}\right)_{+}^{0} \sum_{j=1}^{d_{t}^{\prime}-1} p^{(i+1)}(y)\left(\phi_{t j+1}(y)-\phi_{11}(y)\right)+\mathbf{I}_{1}\left[s^{(i+2)}\right] \\
& =p_{0}^{(i)}(y)+\left(\Phi(y)-\phi_{11}(y)\right) p^{(i+1)}(y)+\mathbf{I}_{1}\left[s^{(i+2)}\right] \\
& =\cdots \cdots \cdots \\
& =p_{0}^{(i)}(y)-\sum_{j=1}^{r-i-1} \frac{(-1)^{j}}{j !}\left(\Phi(y)-\phi_{11}(y)\right)^{j} p^{(i+j)}(y)+\mathbf{I}_{r-i-1}\left[s^{(r)}\right] .
\end{aligned}
$$

This gives

$$
p^{(i)}(y)=\sum_{j=i}^{r-1} \frac{1}{(j-i) !}\left(\Phi(y)-\phi_{11}(y)\right)^{j-i}\left(p_{0}^{(j)}(y)+\mathbf{I}_{r-1-j}\left[s^{(r)}\right]\right) .
$$

Substituting this expression into (17) yields

$$
\begin{aligned}
s(x, y)= & \sum_{i=0}^{r-1} \frac{1}{i !}(x-\Phi)^{i} \sum_{j=i}^{r-1} \frac{1}{(j-i) !}\left(\Phi-\phi_{11}\right)^{j-i}\left(p_{0}^{(j)}+\mathbf{I}_{r-1-j}\left[s^{(r)}\right]\right) \\
& +\int_{\Phi(y)}^{\tau_{0}} \int_{\Phi(y)}^{\tau_{1}} \cdots \int_{\Phi(y)}^{\tau_{r-1}} s^{(r)}\left(\tau_{r}, y\right) d \tau_{r} \cdots d \tau_{2} d \tau_{1} \\
= & \sum_{j=0}^{r-1} \frac{1}{j !}\left(x-\phi_{11}(y)\right)^{j}\left(p_{0}^{(j)}(y)+\mathbf{I}_{r-1-j}\left[s^{(r)}\right]\right) \\
& +\int_{\Phi(y)}^{\tau_{0}} \int_{\Phi(y)}^{\tau_{1}} \cdots \int_{\Phi(y)}^{\tau_{r-1}} s^{(r)}\left(\tau_{r}, y\right) d \tau_{r} \cdots d \tau_{2} d \tau_{1} .
\end{aligned}
$$

Next, we need to discuss the integral conformality condition of $s^{(i)}(x, y), i=$ $1, \ldots, r$, at each interior vertex $v \in V_{l}$. Let $q_{j}^{(i)}(x, y) \in \Pi_{k-r-1}$ be the smoothing 
cofactor of $s^{(i)}(x, y)$ from $T_{j-1}$ to $T_{j}$ across edge $e_{j}$ around $v$; then the integral conformality condition of $s^{(i)}(x, y), i=1, \ldots, r$, at vertex $v$ is equivalent to the system

$$
\left\{\begin{array}{l}
\sum_{j=1}^{d_{v}} \int_{l_{1}(y)}^{l_{j}(y)} q_{j}^{(1)}(x, y)\left[x-l_{j}(y)\right]^{r} d x \equiv 0 \\
\sum_{j=1}^{d_{v}} \int_{l_{1}(y)}^{l_{j}(y)} q_{j}^{(2)}(x, y)\left[x-l_{j}(y)\right]^{r-1} d x \equiv 0 \\
\cdots \cdots \cdots \cdots \\
\sum_{j=1}^{d_{v}} \int_{l_{1}(y)}^{l_{j}(y)} q_{j}^{(r)}(x, y)\left[x-l_{j}(y)\right]^{1} d x \equiv 0 .
\end{array}\right.
$$

From $s^{(i)}(x, y) \in S_{k-i}^{r-i}(\Delta)$, we have, for any $i=1, \ldots, r-1$,

$$
\left.s^{(i)}(x, y)\right|_{\tau_{j}}-\left.s^{(i)}(x, y)\right|_{\tau_{j-1}}=q_{j}^{(i)}(x, y)\left[x-l_{j}(y)\right]^{r-i+1} .
$$

For convenience, we say $T_{j-1}$ is located at the left side of $T_{j}$ if there exists $y^{*}$ such that $\left(x_{j}, y^{*}\right) \in T_{j}$ and $\left(x_{j-1}, y^{*}\right) \in T_{j-1}$ with $x_{j-1}<x_{j}$. Without loss of generality, we can assume that $T_{j-1}$ is located at the left side of $T_{j}$. Then by setting $s_{t}^{(i)}:=\left.s^{(i)}(x, y)\right|_{T_{i}}$, $t=j-1, j$, it follows from (13)-(15) that

$$
\begin{aligned}
s_{j}^{(i)}(x, y)= & p^{(i)}(y)+\int_{\Phi(y)}^{l} s^{(i+1)}(\tau, y) d \tau+\int_{l}^{l_{j}} s_{j-1}^{(i+1)}(\tau, y) d \tau \\
& +\int_{l_{j}}^{x} s_{j}^{(i+1)}(\tau, y) d \tau, \\
s_{j-1}^{(i)}(x, y)= & p^{(i)}(y)+\int_{\Phi(y)}^{l} s^{(i+1)}(\tau, y) d \tau+\int_{l}^{x} s_{j-1}^{(i+1)}(\tau, y) d \tau,
\end{aligned}
$$

where $x=l(y)$ stands for $x=l_{j-1}(y)$ or the equation of the third edge of $T_{j-1}$. These two equalities together with (22) yield

$$
q_{j}^{(i)}(x, y)\left[x-l_{j}(y)\right]^{r-i+1}=\int_{l_{j}}^{x}\left[s_{j}^{(i+1)}(\tau, y)-s_{j-1}^{(i+1)}(\tau, y)\right] d \tau,
$$

that is,

$$
q_{j}^{(i)}(x, y)\left[x-l_{j}(y)\right]^{r-i+1}=\int_{l_{j}}^{x} q_{j}^{(i+1)}(\tau, y)\left[\tau-l_{j}(y)\right]^{r-i} d \tau
$$

Substituting this into the integral-conformality-condition system (21) yields (12). The proof of the theorem is complete. 
Theorem 1 shows that any bivariate spline function $s \in S_{k}^{r}(\Delta)$ can be expressed by a series of univariate polynomials and a special bivariate finite-element function $s^{(r)} \in S_{k-r}^{0}(\Delta)$ satisfying the integral-conformality-condition system (12). Hence all discussion of the space $S_{k}^{r}(\Delta)$ can be transformed into discussion of the integralconformality-condition system (12).

Noticing that, for all $j=0, \ldots, r-1, \mathrm{I}_{r-1-j}\left[s^{(r)}\right]$ is always a univariate polynomial in $\mathbf{P}_{k-j}$, and denoting the subspace of $S_{k-r}^{0}(\triangle)$ with its elements satisfying the integralconformality-condition system (12) by $\tilde{S}_{k-r}^{0}(\Delta)$, we can re-write Theorem 1 .

THEOREM 2. Let $1 \leq r \leq k-1$ and $(\Omega, \Delta)$ be type- $X$; then a necessary and sufficient condition for $s \in S_{k}^{r}(\Delta)$ is that $s$ has the following expansion:

$$
\begin{aligned}
s(x, y)= & \sum_{j=0}^{r-1} \frac{1}{j !}\left(x-\phi_{11}(y)\right)^{j} p_{0}^{(j)}(y) \\
& +\int_{\Phi(y)}^{\tau_{0}} \int_{\Phi(y)}^{\tau_{1}} \cdots \int_{\Phi(y)}^{\tau_{r-1}} s^{(r)}\left(\tau_{r}, y\right) d \tau_{r} \cdots d \tau_{2} d \tau_{1},
\end{aligned}
$$

where $\tau_{0}=x, p_{0}^{(j)}(y)$ are arbitrary univariate polynomials in $\mathbf{P}_{k-j}, j=0, \ldots, r-1$, and $s^{(r)}(x, y) \tilde{S}_{k-r}^{0}(\triangle)$.

In addition, we can easily prove that the expansion in Theorem 2 is unique. Hence we have the following direct sum decomposition of $S_{k}^{r}(\Delta)$.

THEOREM 3. Let $1 \leq r \leq k-1$ and $(\Omega, \Delta)$ be type-X. If we set

$$
\begin{aligned}
\mathbf{W}_{j} & :=\left\{\left(x-\phi_{11}(y)\right)^{j} \cdot p_{0}^{(j)}(y): p_{0}^{(j)} \in \mathbf{P}_{k-j}\right\}, \quad j=0, \ldots, r-1, \\
\mathbf{Q} & :=\left\{\int_{\Phi(y)}^{\tau_{0}} \int_{\Phi(y)}^{\tau_{1}} \cdots \int_{\Phi(y)}^{\tau_{r-1}} s^{(r)}\left(\tau_{r}, y\right) d \tau_{r} \cdots d \tau_{2} d \tau_{1}: s^{(r)} \tilde{S}_{k-r}^{0}(\Delta)\right\},
\end{aligned}
$$

then

$$
S_{k}^{r}(\triangle)=\bigoplus_{j=0}^{r-1} \mathbf{W}_{j} \oplus \mathbf{Q} .
$$

Alternatively, if we note that $\mathbf{Q} \cong \tilde{S}_{k-r}^{0}(\Delta)$, we have the following result.

COROLLARY 1 . Let $1 \leq r \leq k-1$ and $(\Omega, \Delta)$ be type- $X$; then

$$
\operatorname{dim} S_{k}^{r}(\Delta)=\frac{1}{2}(2 k-r+3) r+\operatorname{dim} \tilde{S}_{k-r}^{0}(\Delta)
$$

However, we need to point out that, because the integral-conformality-condition system (12) involves the triangulation $\Delta$, it is still very difficult to discuss, especially for the cases where the smoothness $r$ is close to the degree $k$. This reflects the complexity of the bivariate spline space. 


\section{The bivariate spline space $S_{r+1}^{r}(\Delta)$}

Consider the special case of $k=r+1$. At this time, $s^{(r)}(x, y) \in S_{1}^{0}(\Delta)$ and $q_{j}^{(r)}(x, y) \in \Pi_{0}$, so we can set $c_{j}:=q_{j}^{(r)}(x, y), j=0, \ldots, d_{v}$. Then the integralconformality-condition system (12) can be simplified to

$$
\left\{\begin{array}{l}
\sum_{j=1}^{d_{v}} c_{j}\left[l_{1}(y)-l_{j}(y)\right]^{2} \equiv 0 \\
\sum_{j=1}^{d_{v}} c_{j}\left[l_{1}(y)-l_{j}(y)\right]^{3} \equiv 0 \\
\cdots \cdots \cdots \cdots \\
\sum_{j=1}^{d_{v}} c_{j}\left[l_{1}(y)-l_{j}(y)\right]^{r+1} \equiv 0
\end{array}\right.
$$

that is,

$$
\left\{\begin{array}{l}
\sum_{j=1}^{d_{v}} c_{j}\left[k_{1}^{-1}-k_{j}^{-1}\right]^{2} \equiv 0 \\
\sum_{j=1}^{d_{v}} c_{j}\left[k_{1}^{-1}-k_{j}^{-1}\right]^{3} \equiv 0 \\
\cdots \cdots \cdots \cdots \\
\sum_{j=1}^{d_{v}} c_{j}\left[k_{1}^{-1}-k_{j}^{-1}\right]^{r+1} \equiv 0 .
\end{array}\right.
$$

In addition, note that, for $s^{(r)} \in S_{1}^{0}(\Delta), c_{j}=q_{j}^{(r)}$ should satisfy the conformality condition (2), that is,

$$
\sum_{j=1}^{d_{v}} c_{j}\left[x-l_{j}(y)\right] \equiv 0
$$

which is equivalent to

$$
\left\{\begin{array}{l}
\sum_{j=1}^{d_{v}} c_{j}=0 \\
\sum_{j=1}^{d_{v}} c_{j}\left[k_{1}^{-1}-k_{j}^{-1}\right]=0 .
\end{array}\right.
$$

So, a necessary and sufficient condition for $s \in S_{r+1}^{r}(\Delta)$ is that $s$ can be expanded as (11) with $s^{(r)} \in S_{1}^{0}(\Delta)$ satisfying, at each interior vertex $v \in V_{l}$, the following system:

$$
\left\{\begin{array}{l}
\sum_{j=1}^{d_{v}} c_{j}=0 \\
\sum_{j=1}^{d_{v}}\left[k_{1}^{-1}-k_{j}^{-1}\right] c_{j}=0 \\
\sum_{j=1}^{d_{v}}\left[k_{1}^{-1}-k_{j}^{-1}\right]^{2} c_{j}=0 \\
\cdots \cdots \cdots \cdots \\
\sum_{j=1}^{d_{v}}\left[k_{1}^{-1}-k_{j}^{-1}\right]^{r+1} c_{j}=0 .
\end{array}\right.
$$


The coefficient matrix of the above homogeneous system is

$$
A=\left[\begin{array}{ccccc}
1 & 1 & 1 & \cdots & 1 \\
0 & \lambda_{2} & \lambda_{3} & \cdots & \lambda_{d_{v}} \\
0 & \lambda_{2}^{2} & \lambda_{3}^{2} & \cdots & \lambda_{d_{v}}^{2} \\
\vdots & \vdots & \vdots & \ddots & \vdots \\
0 & \lambda_{2}^{r+1} & \lambda_{3}^{r+1} & \cdots & \lambda_{d_{v}}^{r+1}
\end{array}\right]
$$

with $\lambda_{j}:=k_{1}^{-1}-k_{j}^{-1}, j=2, \ldots, d_{v}$.

It is easy to see that, if $d_{v}=r+2$, then

$$
\operatorname{det} A=\prod_{j=2}^{r+2} \lambda_{j} \cdot \operatorname{det} V\left[\lambda_{2}, \lambda_{3}, \ldots, \lambda_{r+2}\right],
$$

where det $V\left[\lambda_{2}, \lambda_{3}, \ldots, \lambda_{r+2}\right]$ is the well-known Vandermonde determinant. So $\operatorname{det} A \neq 0$ if and only if $k_{i} \neq k_{j}, i, j=1, \ldots, d_{v}, i \neq j$.

We now introduce the following result in which the so-called cross-cut grid line is introduced [25] and defined such that it divides $\Omega$ into two parts.

THEOREM 4. Let $(\Omega, \Delta)$ be type-X and, for each interior vertex $v$, let the number of edges with different slopes attached to $v$ be not greater than $r+2$. Then

$$
\operatorname{dim} S_{r+1}^{r}(\triangle)=\operatorname{dim} \Pi_{r+1}+N_{c},
$$

where $N_{c}$ is the number of all cross-cut grid lines in $\Delta$.

PROOF. First, we just consider the case that $\Delta=\Delta_{v}$. Without loss of generality, we can assume that there are only two interior edges having the same slope, for example, $e_{j_{0}}$ and $e_{1}$. Then the system (32) becomes

$$
\left\{\begin{array}{l}
\sum_{j=1}^{j_{0}-1} c_{j}^{\prime}+\sum_{j=j_{0}+1}^{d_{v}} c_{j}^{\prime}=0 \\
\sum_{j=1}^{j_{0}-1}\left[k_{1}^{-1}-k_{j}^{-1}\right] c_{j}^{\prime}+\sum_{j=j_{v}+1}^{d_{v}}\left[k_{1}^{-1}-k_{j}^{-1}\right] c_{j}^{\prime}=0 \\
\sum_{j=1}^{j_{0}-1}\left[k_{1}^{-1}-k_{j}^{-1}\right]^{2} c_{j}^{\prime}+\sum_{j=j_{0}+1}^{d_{v}}\left[k_{1}^{-1}-k_{j}^{-1}\right]^{2} c_{j}^{\prime}=0 \\
\cdots \cdots \cdots \cdots \\
\sum_{j=1}^{j_{0}-1}\left[k_{1}^{-1}-k_{j}^{-1}\right]^{r+1} c_{j}^{\prime}+\sum_{j=j_{0}+1}^{d_{v}}\left[k_{1}^{-1}-k_{j}^{-1}\right]^{r+1} c_{j}^{\prime}=0,
\end{array}\right.
$$

with $c_{1}^{\prime}=c_{1}+c_{j_{0}}$ and $c_{j}^{\prime}=c_{j}, j=2, \ldots, d_{v}, j \neq j_{0}$. Since the number of edges with different slopes attached to $v$ is not greater than $r+2$, that is, $d_{v}-1 \leq r+2$, the system (36) has a unique zero solution:

$$
c_{1}^{\prime}=c_{2}^{\prime}=\cdots=c_{j_{0}-1}^{\prime}=c_{j_{0}+1}^{\prime}=\cdots=c_{d_{v}}^{\prime}=0,
$$


that is,

$$
c_{1}=-c_{j_{0}}, \quad c_{2}=\cdots=c_{j_{0}-1}=c_{j_{0}+1}=\cdots=c_{d_{v}}=0 .
$$

Hence the dimension of $\tilde{S}_{1}^{0}(\Delta)$ equals the sum of the dimension, 3, contributed from the so-called source-cell $[8,9,25]$ and the dimension, 1 , contributed from all the smoothing cofactors. Furthermore, according to Corollary 1 , we can easily have

$$
\operatorname{dim} S_{r+1}^{r}(\Delta)=\frac{1}{2}(r+5) r+4=\operatorname{dim} \Pi_{r+1}+1=\operatorname{dim} \Pi_{r+1}+N_{c} .
$$

Secondly, we consider the case that the number of interior vertices in $\Delta$ is more than 1. Without loss of generality, we can still assume that there is only one cross-cut grid line, denoted by $L$ in $\triangle$. From one end to the other, we denote all vertices which lie on $L$ by $v^{(i)}, i=0,1, \ldots, N+1$, as shown in Figure 2, where $v^{(0)}$ and $v^{(N+1)}$ are two boundary vertices. For each $v^{(i)}, i=1, \ldots, N$, we denote all the edges attached to $v^{(i)}$ in the counter-clockwise direction by $e_{j}^{(i)}, j=1, \ldots, d_{i}$, where $d_{i}$ is the degree of $v^{(i)}$. Without loss of generality, we can assume that $e_{1}^{(i)}$ and $e_{j_{i}}^{(i)}$ lie on the cross-cut grid line $L$ and the corresponding smoothing cofactors of $s^{(r)} \in S_{1}^{0}(\Delta)$ across $e_{1}^{(i)}$ and $e_{j_{i}}^{(i)}$ around $v^{(i)}$ are denoted by $c_{1}^{(i)}$ and $c_{j_{i}}^{(i)}$, respectively. Then from the proof of the first case, we have

$$
c_{1}^{(i)}=-c_{j_{i}}^{(i)}, \quad i=1, \ldots, N
$$

Note that $c_{j_{i}}^{(i+1)}=-c_{1}^{(i)}[8,9,25]$ and we have

$$
c_{1}^{(i)}=c_{1}^{(1)}, \quad i=1, \ldots, N .
$$

Now, let $v$ be any interior vertex in $V_{I}$. Since the number of edges with different slopes attached to $v$ is not greater than $r+2$, it follows from the proof of the first case that, for any edge $e_{j}$ attached to $v$, if no other edge has the same slope, then the corresponding smoothing cofactor $c_{j}=0$; otherwise $c_{j}=-c_{j_{0}}$, where $e_{j_{0}}$ has the same slope as edge $e_{j}$. For the latter case, $e_{j}$ and $e_{j_{0}}$ either lie on the unique cross-cut grid line $L$ or a line segment $L^{*}$. We have proved that if they lie on $L$ then both $c_{j}$ and $c_{j_{0}}$ are determined by $c_{1}^{(1)}$. Hence we need merely discuss the case that $e_{j}$ and $e_{j_{0}}$ lie on the line segment $L^{*}$.

Because $L^{*}$ is not a cross-cut grid line, there exists at least one end point of $L^{*}$ that is an interior vertex of $\Delta$, denoted by $v^{*}$, as shown in Figure 2 . From this end point $v^{*}$ to $v$, we denote all vertices that lie on $L^{*}$ by $v_{j}^{*}, j=0,1, \ldots, N^{*}$, with $v^{*}=v_{0}^{*}$ and $v=v_{N^{*}}^{*}$. Similarly to (41), we conclude that each smoothing cofactor of $s^{(r)}(x, y)$ across edge $v_{j}^{*} v_{j+1}^{*}$ around $v_{j+1}^{*}, j=0,1, \ldots, N^{*}-1$, equals the smoothing cofactor of $s^{(r)}(x, y)$ across edge $v_{0}^{*} v_{1}^{*}$ around $v_{1}^{*}$. Since there is no other interior edge in $\triangle_{v_{0}^{*}}$ 


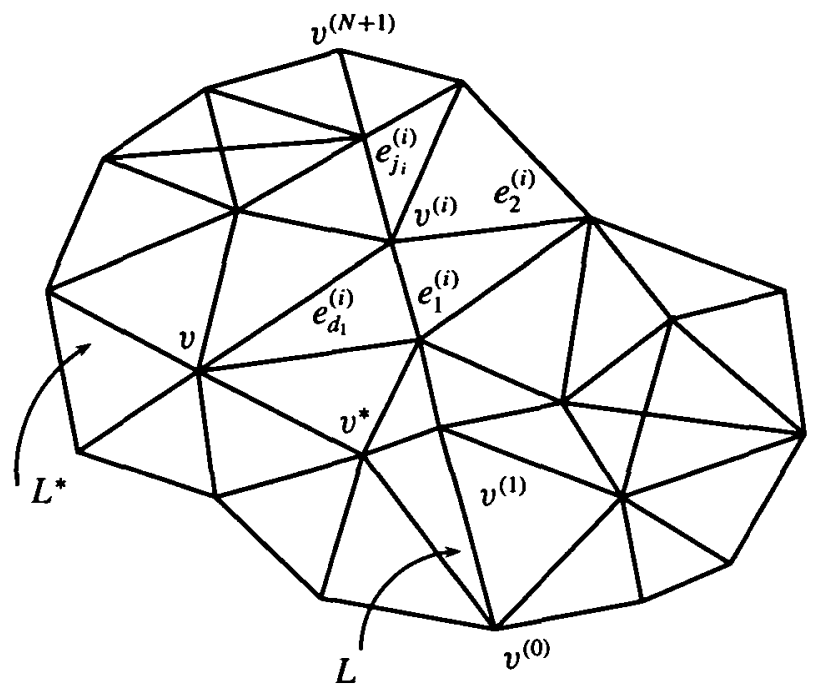

FIGURE 2. A particular triangulation $\Delta$ of $\Omega$ in Theorem 4 .

having the same slope as edge $v_{0}^{*} v_{1}^{*}$, it follows from the proof of the first case that the smoothing cofactor of $s^{(r)}(x, y)$ across edge $v_{0}^{*} v_{1}^{*}$ around $v_{1}^{*}$ equals 0 . So all the smoothing cofactors of $s^{(r)}(x, y)$ across all the edges that lie on $L^{*}$ are equal to 0 , meaning $c_{j}=0$.

So we conclude that the dimension contributed by the edges lying on the cross-cut grid line $L$ is 1 and by other edges is 0 . This results in the same result as (39). The proof of the theorem is completed.

It is worth indicating that, in the special case discussed in Theorem 4, our dimension result is not the lower bound of the dimension formula given by Schumaker [24]. In fact, if we still use $e_{i}$, as in [24], to stand for the number of edges with different slopes attached to the $i$ th interior vertex, $i=1,2, \ldots,\left|V_{l}\right|$, then [24] gives

$$
\begin{aligned}
\operatorname{dim} S_{r+1}^{r}(\Delta) & \geq \frac{1}{2}(r+2)(r+3)+\left|E_{I}\right|-(r+2)\left|V_{I}\right|+\sum_{i=1}^{\left|V_{I}\right|}\left(r+2-e_{i}\right)_{+} \\
& =\frac{1}{2}(r+2)(r+3)+\left|E_{I}\right|-(r+2)\left|V_{I}\right|+\sum_{i=1}^{\left|V_{I}\right|}\left(r+2-e_{i}\right) \\
& =\frac{1}{2}(r+2)(r+3)+\left|E_{I}\right|-(r+2)\left|V_{I}\right|-\sum_{i=1}^{\left|V_{I}\right|} e_{i} .
\end{aligned}
$$

Generally speaking, the lower bound (42) is often smaller than the dimension given 
in (35).

COROLLARY 2. Let $(\Omega, \Delta)$ be type-X and, for each interior vertex $v$, let the number of all edges around $v$ with different slopes be not greater than $r+2$. If $N_{c}=0$ then the spline space $S_{r+1}^{r}(\Delta)$ degenerates to a trivial polynomial space $\Pi_{r+1}$.

From Corollary 1 we can easily see that, for the well-known Morgan-Scott triangulation $\Delta_{s}$ [23], whether it is symmetrical or not, the bivariate spline space $S_{r+1}^{r}(\Delta)$ is always a trivial polynomial space $\Pi_{r+1}$ when $r \geq 2$.

\section{Acknowledgements}

The author would like to gratefully acknowledge financial support from DEET Australia an Overseas Postgraduate Research Scholarship and from the University of Wollongong (a University Postgraduate Award) in carrying out this research project. $\mathrm{He}$ is also obliged to Professor Jim Hill, the editor and the referee for their valuable suggestions. Finally, his thanks are also given to Dr Ahmed El-Feki for carefully reading the original version of this paper.

\section{References}

[1] P. Alfeld, M. Neamtu and L. L. Schumaker, "Fitting scattered data on sphere-like surfaces using spherical splines", J. Comp. Appl. Math. 73 (1996) 5-43.

[2] P. Alfeld, B. Piper and L. L. Schumaker, "An explicit basis for $C^{\prime}$ quartic bivariate splines", SIAM J. Numer. Anal. 24 (1987) 891-911.

[3] P. Alfeld and L. L. Schumaker, "The dimension of bivariate spline spaces of smoothness $r$ for degree $d \geq 4 r+1$ ", Const. Approx. 3 (1987) 189-197.

[4] C. de Boor, "B-form basis", in Geometric Modeling (ed. G. Farin), (SIAM, Philadelphia, 1987) 21-28.

[5] C. de Boor, K. Hollig, S. Riemenschneider and A. Ron (eds.), Box splines (Springer-Verlag, New York, 1993).

[6] C. K. Chui and J. Z. Wang, "On compactly supported spline wavelets and a duality principle", Trans. Amer. Math. Society 330 (1992) 903-916.

[7] C. K. Chui and J. Z. Wang, "A cardinal spline approach to wavelets", Proc. Amer. Math. Society 113 (1991) 785-793.

[8] C. K. Chui and R. H. Wang, "Multivariate spline spaces", J. Math. Anal. Appl. 94 (1983) 197-221.

[9] C. K. Chui and R. H. Wang, "On smooth multivariate spline functions", Math. Comp. 47 (1983) $131-142$.

[10] W. Dahmen and C. A. Micchelli, "Recent progress in multivariate splines", in Approximation theory IV (eds. C. K. Chui, L. L. Schumaker and J. Ward) (Academic Press, 1983) 27-121.

[11] G. Farin, "Triangular Bernstein-Bézier patches", Computer Aided Geometric Design 3 (1986) 87-127. 
[12] G. Farin (eds.), Curves and surfaces for computer aided geometric design, a practical guide (Academic Press, New York, 1988).

[13] D. Hong, "Spaces of bivariate spline functions over triangulations", Approx. Theory and Appl. 7 (1991) 56-75.

[14] D. Hong, "A new formulation of Bernstein-Bézier based smoothness conditions for $p p$ functions", Approx. Theory and Appl. 11 (1995) 67-75.

[15] D. Hong and H.-W. Liu and Ram Mohapatra, "Optimal triangulations and smoothness conditions for bivariate splines", in Approximation theory IX, Vol. 2: Computational Aspects (eds. C. K. Chui, L. L. Schumaker), (Vanderbilt University Press, Nashville, 1998) 129-136.

[16] R. Q. Jia, "B-net representation of multivariate splines", Ke Xue Tong Bao (A Monthly Journal of Science) 11 (1987) 804-807.

[17] R. Q. Jia, "A dual basis for the integer translates of an exponential box spline", The Rocky Mountain Journal of Mathematics 23 (1993) 223-242.

[18] Y. S. Li and L. T. Guan, "Bivariate polynomial natural spline interpolation to scattered data", J. Comp. Math.: An International Journal on Numerical Methods, Analysis and Applications 8 (1990) 135-146.

[19] H.-W. Liu, "The double periodic spline space with degree $k \geq 4$ on Type-1 triangulation", CALCOLO 29 (1992) 269-289.

[20] H. W. Liu, "An integral representation of bivariate splines and the dimension of quadratic spline spaces over stratified triangulation", Acta Math. Sinica 4 (1994) 534-543.

[21] H.-W. Liu, "The dimension of cubic spline space over stratified triangulation", J. Math. Research. and Exposition 16 (1996) 199-208.

[22] J. Morgan and R. Scott, "A nodal basis for $C^{1}$ piecewise polynomials of degree $n \geq 5$ ", Math. Comp. 29 (1975) 736-740.

[23] J. Morgan and R. Scott, "The dimension of piecewise polynomials", manuscript.

[24] L. L. Schumaker, "On the dimension of spaces of piecewise polynomials in two variables", in Multivariable Approximation Theory (eds. W. Schempp and K. Zeller), (Birkhäuser, Basel, 1979) 396-412.

[25] R. H. Wang, "The structural characterization and interpolation for multivariate splines", Acta. Math. Sinica 18 (1975), 91-106.

[26] P. Zwart, "Multivariate splines with non-degenerate partitions", SIAM J. Numer. Anal. 10 (1973) 665-673. 